\title{
Modeling and Analysis of the Effect of Dip-Spin Coating Process Parameters on Coating Thickness Using Factorial Design Method
}

\author{
Sorasutee Buapool, Nandh Thavarungkul, Nakorn Srisukhumbowornchai, \\ and Preecha Termsuksawad
}

Integrated Product Design and Manufacturing Program, Division of Materials Technology, School of Energy, Environment and Materials, King Mongkut's University of Technology Thonburi, 126 Pracha Uthit Road, Bang Mod, Thung Khru, Bangkok 10140, Thailand

Correspondence should be addressed to Sorasutee Buapool; sorasutee@gmail.com

Received 1 January 2017; Accepted 22 March 2017; Published 23 April 2017

Academic Editor: Luciano Lamberti

Copyright (C) 2017 Sorasutee Buapool et al. This is an open access article distributed under the Creative Commons Attribution License, which permits unrestricted use, distribution, and reproduction in any medium, provided the original work is properly cited.

\begin{abstract}
Statistical modeling of the dip-spin coating process to describe colloidal PTFE dispersion coating on the external surface of a small diameter hollow tube was developed by using $2^{4}$ factorial design with a center point to predict the coating thickness in a range of $4-10 \mu \mathrm{m}$. The coating parameters included viscosity, withdrawal rate, spin speed, and immersion time. The adequacy of the predicted model was verified by coefficients of determination and lack-of-fit test. Model accuracy was verified by comparing predicted values with experimental results. The significant interaction effects on the coating thickness were three-way interaction among withdrawal rate, spin speed, and immersion time and two-way interactions between viscosity and withdrawal rate, viscosity and spin speed, and viscosity and immersion time. Cube plot for coating thickness reveals a trend of increasing coating thickness towards high levels of viscosity, withdrawal rate, and immersion time and lower level of spin speed.
\end{abstract}

\section{Introduction}

Fluoropolymer dispersion coating is commonly used for nonstick coating applications that require friction-reducing property and electrical insulation, adhere to biosafety standards, and display chemical resistance and wear resistance [1]. This process is utilized in industry-level production by spraying, dip coating, dip-spin coating, and curtain coating processes [2]. Contrary to statistical models of wide-ranging applications, these processes have very limited appropriate statistical models to predict coating process parameters and their property relationships. To overcome extensive experimental setup and uncontrollable parameters that might affect the coating of fluoropolymer dispersion, programmable dipspin coating equipment was newly constructed for improved process control. Polytetrafluoroethylene (PTFE) dispersion is an example of fluoropolymer dispersion used in this study. Due to its colloid nature in volatile components, the dip-spin coating equipment will disperse insoluble polymer particles in a continuous medium and have features of dip coating, spinning, heating, and blowing pressurized air.

In this study, the exterior coating on a stainless steel short hollow tube with very small diameter is of great interest for its highly curved surface, which is used to determine whether the nonstick PTFE dispersion could be coated by this dipspin coating equipment. Good coating properties of the films such as film thickness and uniformity are examined to reflect the processability of this equipment. The basic principle of dip coating is to deposit thin films of polymer solution on a substrate by immersing the substrate in a polymer solution for some time and then withdrawing the substrate from the polymer solution bath [3]. Many of the previous studies of dip coating processes [4-10] were designed to determine the effect of coating process parameters on the layer thickness. The layer thickness depends on, for example, withdrawal rate, number of dip cycles, and physical properties of the fluid. 


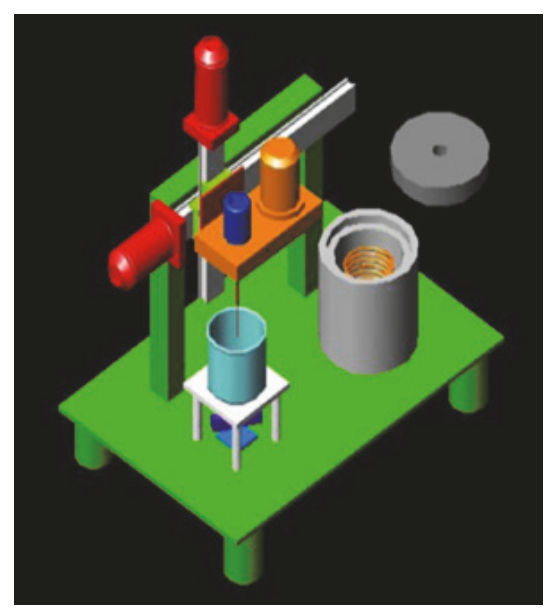

(a)

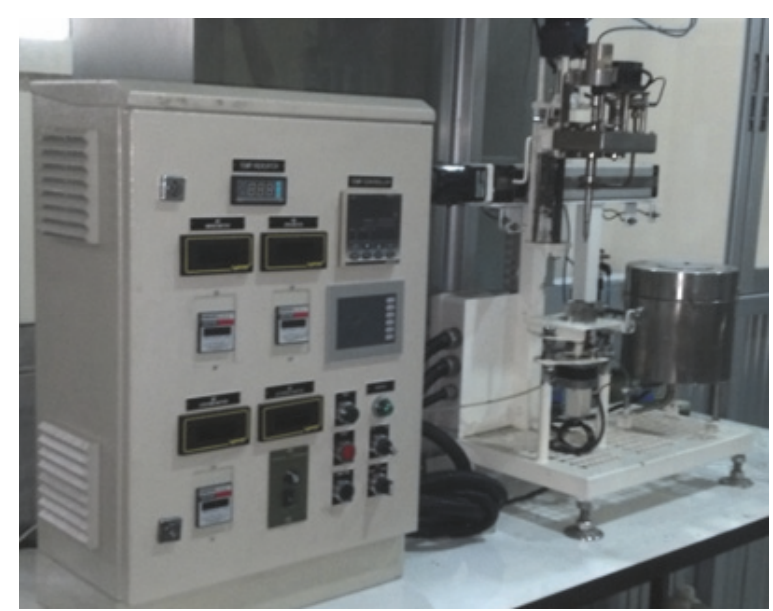

(b)

FIGURE 1: (a) Schematic diagram and (b) actual prototype of the dip-spin coating equipment.

The mathematical models represent the fluid dynamics of a dip coating process for materials with a Newtonian fluid and non-Newtonian fluid behavior. An early theoretical model for dip coating analysis presented by Landau and Levich [11] and Derjaguin [12] (LLD) was based on a Newtonian fluid flow without considering solvent evaporation. The relationship between film thickness $(h)$ and withdrawal rate $(v)$ was estimated by the power law of the form $h \propto v^{x}$, where the value of $x$ was the power law index obtained from the experiment. Furthermore, addition of centrifugal force from spinning right after withdrawing the substrate from the polymer solution bath helps to remove excess polymer from the outer surface and improve coating uniformity.

As several independent variables affect polymer coating, the aim of this study was, therefore, to determine the main and interaction effects of the coating parameters on PTFE thickness by using the programmable dip-spin coating equipment. The factorial design was used to design the experiments and analyze the results. A design of experiment methodology can reduce the number of experiments by studying multiple factors. The relationship reflecting PTFE coating thickness was investigated by using a $2^{4}$ factorial design with a center point. The factorial design was to examine the main and interaction effects of the coating parameters and to establish an empirical model to predict the coating thickness. The interaction effect is defined as the dependence of the effect of one independent parameter on the level of the other parameter [13]. The coating parameters consisted of viscosity, withdrawal rate, spin speed, and immersion time in the polymer solution. The thickness targets were set in a range of $4-10 \mu \mathrm{m}$.

\section{Experimental}

\subsection{Materials and Methods}

2.1.1. The Dip-Spin Coating Equipment. The newly designed dip-spin coating equipment is a tabletop type with dimensions of about $0.6 \times 1 \mathrm{~m}^{2}$ and is $1 \mathrm{~m}$ tall and consists of the following key components: a substrate holder unit, a polymer dispersion mixer, an oven, a motor driven positioning system, and a control system. In this study, this equipment was used to coat PTFE dispersion on the exterior of a stainless steel short hollow tube, but not on the interior of the tube. To remove the polymer solution out of the bore, an air pressure line installed inside the substrate holder could flow pressurized air through the tube up to $1000 \mathrm{kPa}$.

In order to easily program and control all movement directions and speeds of DC servo motors, all coating process sequences were programmed by using a programmable logic controller (PLC) at the control panel. The program settings included holder movement directions and speeds, pressure of air flowing through the holder, stirring speed at the polymer solution bath, and oven temperature and heating time. The holder could move upwards and downwards at constant speeds up to $25 \mathrm{~mm} / \mathrm{s}$ and spin either clockwise or counterclockwise at speeds up to $720 \mathrm{rpm}$. The holder can move left and right between the polymer solution tank and the oven at speeds up to $40 \mathrm{~mm} / \mathrm{s}$. The stirring speeds at the polymer solution bath could be set in a range of $0-300 \mathrm{rpm}$. The oven could be programmed in four individual segments, each with temperatures up to $800^{\circ} \mathrm{C}$ and time intervals up to 99 hours and 59 minutes.

A schematic diagram and an actual prototype of the dipspin equipment are shown in Figure 1. For one complete cycle of the dip-spin coating process, a short hollow tube substrate was immersed into the polymer solution bath. After some required immersion time in the bath, the substrate was withdrawn at the same rate as its immersion. Right after withdrawing from the polymer tank, the substrate was spun for $5 \mathrm{~s}$ each in clockwise and counterclockwise directions to remove excess polymer on the outer surface. In the meantime, pressurized air applied at $400 \mathrm{kPa}$ flew through the bore to remove excess polymer remaining inside the tube. The substrate was then carried within a few seconds to the oven where film formation on the outer surface took place. The curing temperature and time were set accordingly 
TABLE 1: Coating parameters used in the $2^{k}$ factorial experiments.

\begin{tabular}{lcccccc}
\hline Parameters & Units & \multicolumn{3}{c}{ Levels (uncoded) } & \multicolumn{2}{c}{ Levels (coded) } \\
\hline Viscosity $(A)$ & $\mathrm{mPa} \cdot \mathrm{s}$ & 900 & 1000 & 1100 & -1 & 0 \\
Withdrawal rate $(B)$ & $\mathrm{mm} / \mathrm{s}$ & 20 & 22.5 & 25 & -1 & 0 \\
Spin speed $(C)$ & $\mathrm{rpm}$ & 100 & 300 & 500 & -1 & +1 \\
Immersion time $(D)$ & $\mathrm{s}$ & 10 & 15 & 20 & -1 & 0 \\
\hline
\end{tabular}

TABLE 2: Design matrix and corresponding results.

\begin{tabular}{|c|c|c|c|c|c|c|c|}
\hline \multirow{2}{*}{$A$} & \multirow{2}{*}{$B$} & \multirow{2}{*}{$C$} & \multirow{2}{*}{$D$} & \multicolumn{3}{|c|}{ Replicate } & \multirow{2}{*}{$\begin{array}{l}\text { Coating thickness } \\
\text { (average } \pm \mathrm{SD})(\mu \mathrm{m})\end{array}$} \\
\hline & & & & 1 & 2 & 3 & \\
\hline \multirow{8}{*}{900} & \multirow{4}{*}{20} & \multirow{2}{*}{100} & 10 & 4.79 & 4.80 & 4.79 & $4.79 \pm 0.01$ \\
\hline & & & 20 & 5.28 & 5.33 & 5.34 & $5.32 \pm 0.03$ \\
\hline & & \multirow{2}{*}{500} & 10 & 4.69 & 4.64 & 4.56 & $4.63 \pm 0.07$ \\
\hline & & & 20 & 4.96 & 4.98 & 5.02 & $4.99 \pm 0.03$ \\
\hline & \multirow{4}{*}{25} & \multirow{2}{*}{100} & 10 & 5.53 & 5.30 & 5.26 & $5.36 \pm 0.15$ \\
\hline & & & 20 & 5.81 & 5.80 & 5.79 & $5.80 \pm 0.01$ \\
\hline & & \multirow{2}{*}{500} & 10 & 5.21 & 5.05 & 4.99 & $5.08 \pm 0.11$ \\
\hline & & & 20 & 5.77 & 5.46 & 5.60 & $5.61 \pm 0.16$ \\
\hline \multirow{8}{*}{1100} & \multirow{4}{*}{20} & \multirow{2}{*}{100} & 10 & 6.02 & 6.22 & 6.49 & $6.24 \pm 0.24$ \\
\hline & & & 20 & 7.27 & 7.09 & 7.26 & $7.21 \pm 0.10$ \\
\hline & & \multirow{2}{*}{500} & 10 & 5.51 & 6.08 & 6.03 & $5.87 \pm 0.32$ \\
\hline & & & 20 & 6.28 & 6.38 & 6.55 & $6.40 \pm 0.14$ \\
\hline & \multirow{4}{*}{25} & \multirow{2}{*}{100} & 10 & 7.17 & 7.42 & 6.77 & $7.12 \pm 0.33$ \\
\hline & & & 20 & 7.88 & 7.91 & 8.18 & $7.99 \pm 0.17$ \\
\hline & & \multirow{2}{*}{500} & 10 & 6.85 & 6.59 & 6.14 & $6.53 \pm 0.36$ \\
\hline & & & 20 & 7.68 & 7.61 & 7.61 & $7.63 \pm 0.04$ \\
\hline 1000 & 22.5 & 300 & 15 & \multicolumn{3}{|c|}{$6.10,5.95,5.95,6.28,6.15$} & $6.09 \pm 0.13$ \\
\hline
\end{tabular}

to the recommended polymer coating factsheet at $400^{\circ} \mathrm{C}$ for $3 \mathrm{~min}$. Preliminary experiments revealed that, for curing temperatures lower than $400^{\circ} \mathrm{C}$, coating cannot form a film on the substrate. For curing temperatures higher than $400^{\circ} \mathrm{C}$, coating has poor quality (i.e., color changes to dark brown and starts degrading). As far as curing time is concerned, films do not form for shorter curing times while longer times have the same effect of higher curing temperatures. Evaporation of solvents could bring polymer particles closer together and interdiffusion of polymer molecules across the substrate could form a film. The coating environment was controlled at about $25^{\circ} \mathrm{C}$ and $60 \%$ relative humidity.

The process parameters for this investigation were selected as shown in Table 1.

\subsubsection{Materials}

(1) Coating Material. The coating material used in this study was premixed polymer solution of PTFE dispersion. The polymer solution included colloidal PTFE particles, polyethersulfone (PES), titanium dioxide pigment, and solvents, which were mainly methyl isobutyl ketone (MIBK) and N-methyl-2-pyrrolidone (NMP) [2]. When the polymer solution was stored, polymer particles slowly settled at the bottom of the container. To restore dispersion uniformity and viscosity prior to coating application of each run as shown in Table 2, the container of the polymer solution was placed on a roller mixer to scatter the sediment and redistribute the polymer particles in a continuous medium. The roller mixer was a separate unit, not shown in Figure 1(a). MIBK and NMP solution mixture was used to adjust viscosities. Polymer agglomerates and other contaminants were strained using wire sieves. Before the polymer solution was loaded to the polymer solution bath located on top of the polymer dispersion mixer, the polymer solution was tested for its viscosity. The viscosity test was conducted following the ASTM D2196-10 standard [14] by using a rotational Brookfield LV viscometer with spindle No. 2 at $20 \mathrm{rpm}$ and $25^{\circ} \mathrm{C}$. The viscosities for the investigation were assessed as in Table 1.

(2) Substrates. The substrates used in this study were commercially available 316 stainless steel short hollow tubes with length and diameter of $4 \mathrm{~mm}$ and $450 \mu \mathrm{m}$, respectively. Substrate preparation was to remove surface contaminants by using a standard cleansing solution and air drying [15]. 


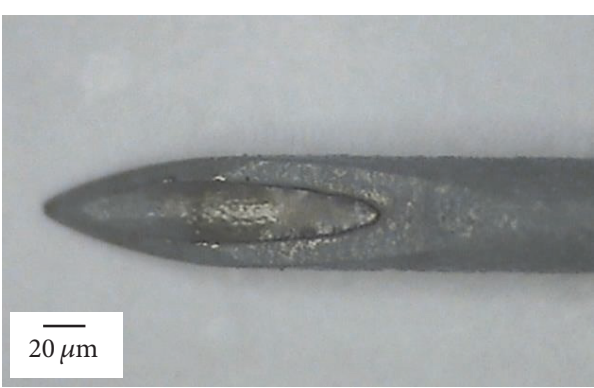

(a)

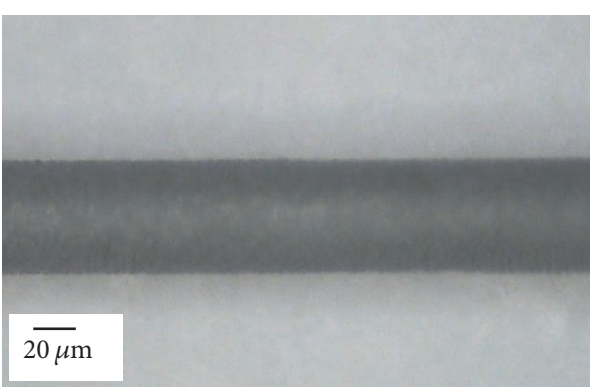

(b)

FIGURE 2: Optical micrographs of the substrate surface (a) at the end and (b) along the short hollow tube.

2.2. Inspection of Coating Thickness. Optical microscopy was used to inspect the coating and measure the coating thickness. Sample preparation included cold mounting using low shrinkage and low viscosity epoxy resin, cross-sectioning at $15 \mathrm{~mm}$ from the end of the hollow tube, and grinding and polishing to fine finish prior to examination.

2.3. Experimental Design. A $2^{4}$ factorial design with a center point was used to evaluate the effects of polymer viscosity $(A)$, withdrawal rate $(B)$, spin speed $(C)$, and immersion time $(D)$ on the coating thickness. The uncoded and coded levels of all coating parameters are shown in Table 1.

The coded levels of each parameter are calculated by the following expression:

$$
x_{\text {Coded }}=\frac{X_{\text {Uncoded }}-X_{\text {center }}}{X_{\text {high }}-X_{\text {center }}},
$$

where $X_{\text {high }}$ is the uncoded value at the upper level of the parameter, $X_{\text {center }}$ is the uncoded level at the center point of the parameter, and $x_{\text {Coded }}$ is the coded value of the parameter of specific value of $X[13]$. The investigated coded values at the lower level, the center point, and the higher level were, respectively, designated as $-1,0$, and +1 .

The design matrix of coating parameters and the corresponding output are shown in Table 2. Parameters were selected so as to produce coatings with thickness in the 4-10micron range. Each run was carried out with three replicates at factorial points but five replicates at the center point. The results of the experiment were statistically analyzed using Minitab $^{\circledR} 16$ software. The statistical model can be used to predict the response parameter, $Y_{i}$, for each coating parameter condition can be described by the following equation:

$$
\begin{aligned}
Y_{i}= & \beta_{0}+\beta_{1} X_{1}+\beta_{2} X_{2}+\beta_{3} X_{3}+\beta_{4} X_{4}+\beta_{12} X_{1} X_{2} \\
& +\beta_{13} X_{1} X_{3}+\beta_{14} X_{1} X_{4}+\beta_{23} X_{2} X_{3}+\beta_{24} X_{2} X_{4} \\
& +\beta_{123} X_{1} X_{2} X_{3}+\beta_{124} X_{1} X_{2} X_{4}+\beta_{234} X_{2} X_{3} X_{4} \\
& +\beta_{1234} X_{1} X_{2} X_{3} X_{4}+\varepsilon,
\end{aligned}
$$

where $Y_{i}$ is the coating thickness, $X_{i}$ are the values of parameters, $\beta_{0}$ is a constant, $\beta_{i}$ is the coefficients of each parameter, $\beta_{i j}, \beta_{i j k}$, and $\beta_{i j k l}(i j k l=1,2,3,4)$ are the coefficients of two-way interactions, three-way interactions, and four-way interactions, respectively, and $\varepsilon$ is a random error term [13]. This model can be effectively used to predict the coating thickness within the ranges of parameters.

\section{Results and Discussion}

3.1. Analysis of Coating Characteristics. The surface of the small hollow tube was successfully coated by using this dipspin coating equipment. The surface of coating, shown in Figure 2, is smooth and does not have polymer blob, mud cracks, stress cracks, wrinkling, or cratering. Compressed air flowing through the tube successfully removed the PTFE polymer inside the bore and left no residual at the distal end of the tube (Figure 2(a)). As a result, the overall surface quality of the coatings on the short hollow tube substrates was satisfactory. Micrographs of the cross sections, Figures 3-5, reveal good adhesive coating of the colloidal PTFE films on substrates of the small hollow tubes. Some phase contrast areas of PES and pigment are also visible in the films along with some dark contrast appearance of epoxy mount interface.

In the dip coating process, the layer of polymer solution on the surface took place after immersing and withdrawing the substrate from the polymer solution tank. At longer immersion times, more polymer particles adsorbing onto the substrate surface had an opportunity to orient on the substrate and reach equilibrium between entraining and receding particles in the tank [16]. Thickness of the polymer film on the substrate depended on the viscous drag holding polymer solution on the substrate during drawing the substrate at a given velocity upwards from the tank against the gravity draining of the solution and, in addition, evaporation of the solvent [16]. Controlled withdrawal rate is crucial to polymer layer thickness forming as a result of force balancing between the viscous drag, the gravity force, and surface tension. The viscous drag is the force moving the liquid upwards with the substrate and is proportional to polymer viscosity and withdrawal rate. Gravity acts to drive the polymer solution downwards. Surface curvature induced by surface tension produces driving force in the same direction as gravity. At given conditions of polymer solution viscosity and spinning speed, the average coating thickness of polymer films increases with withdrawal rates and immersion 


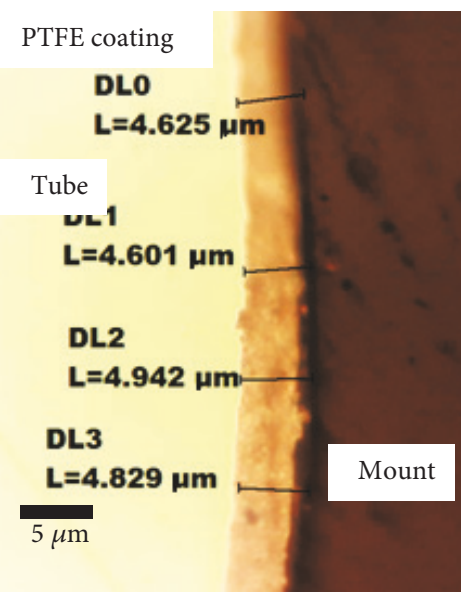

(a)

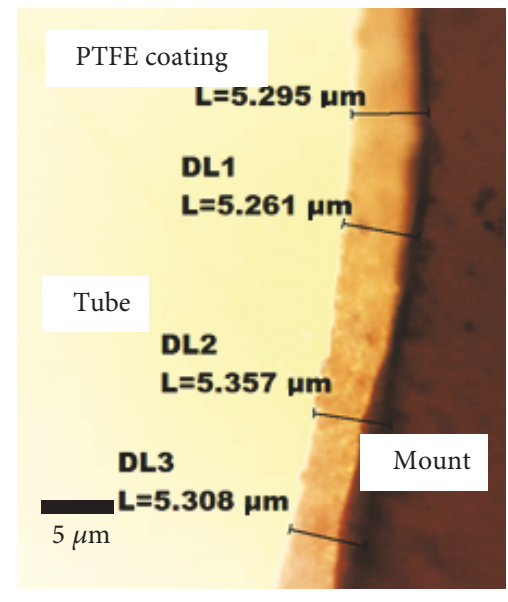

(b)

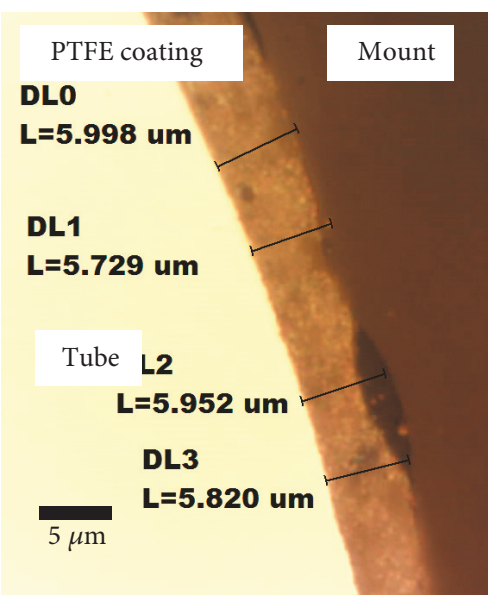

(d)

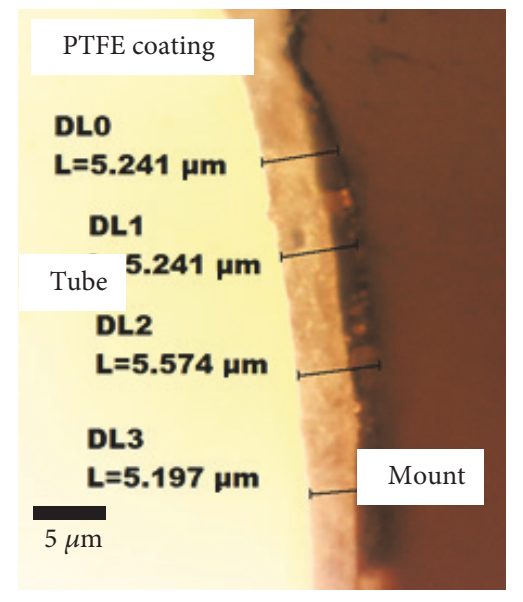

(c) $900 \mathrm{mPa} \cdot \mathrm{s}$, withdrawal rate of $20 \mathrm{~mm} / \mathrm{s}$, and spin speed of $100 \mathrm{rpm}$ ) and (c) $10 \mathrm{~s}$ and (d) $20 \mathrm{~s}$ (viscosity of $900 \mathrm{mPa} \cdot \mathrm{s}$, withdrawal rate of $25 \mathrm{~mm} / \mathrm{s}$, and spin speed of $100 \mathrm{rpm}$ )

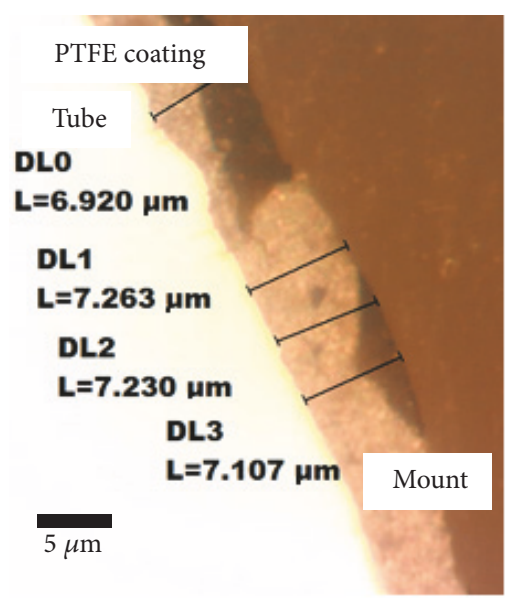

(a)

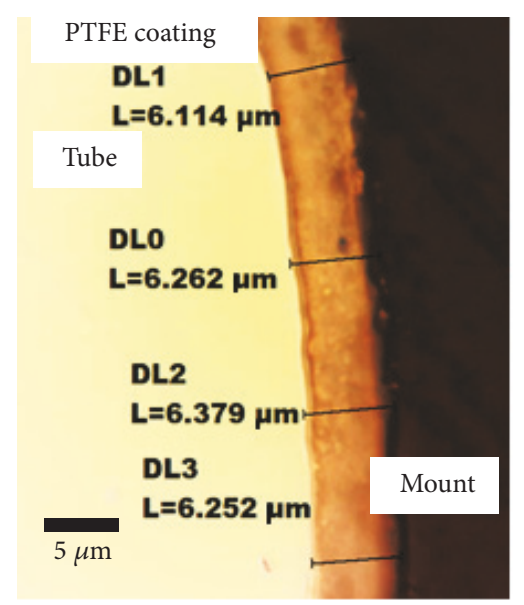

(b)

Figure 4: Optical micrographs of the cross-sectional area of the sample at different spin speeds: (a) $100 \mathrm{rpm}$ and (b) $500 \mathrm{rpm}$ (viscosity of $1100 \mathrm{mPa} \cdot \mathrm{s}$, withdrawal rate of $20 \mathrm{~mm} / \mathrm{s}$, and immersion time of $20 \mathrm{~s}$ ). 


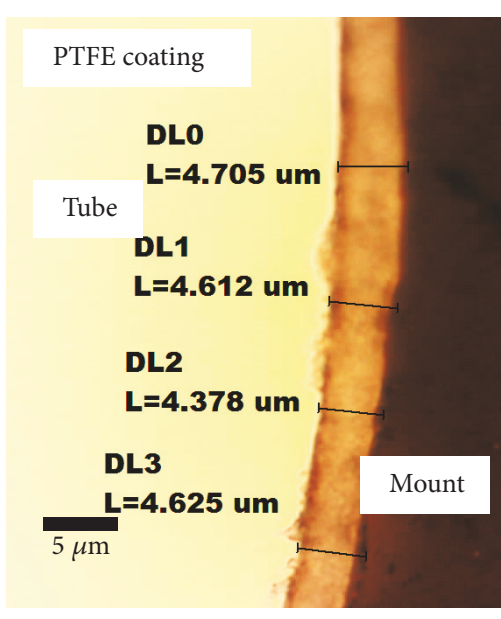

(a)

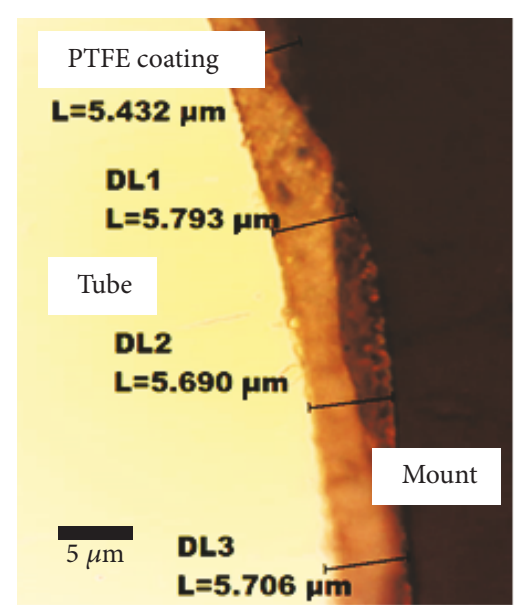

(b)

FIGURE 5: Optical micrographs of the cross-sectional area of the sample at different viscosities: (a) $900 \mathrm{mPa} \cdot \mathrm{s}$ and (b) $1100 \mathrm{mPa} \cdot \mathrm{s}$ (withdrawal rate of $20 \mathrm{~mm} / \mathrm{s}$, spin speed of $500 \mathrm{rpm}$, and immersion time of $10 \mathrm{~s}$ ).

times. When the substrates were coated with a polymer solution having viscosity of $900 \mathrm{mPa} \cdot \mathrm{s}$ and spun at speed of $100 \mathrm{rpm}$, for example, the average coating thicknesses of the films withdrawn at the rate of $20 \mathrm{~mm} / \mathrm{s}$ increased from about $4.79 \mu \mathrm{m}$ to about $5.32 \mu \mathrm{m}$ as immersion time increased from $10 \mathrm{~s}$ to $20 \mathrm{~s}$, respectively, shown in Figures 3(a) and 3 (b). When the withdrawal rates increased to $25 \mathrm{~mm} / \mathrm{s}$, the average coating thicknesses were, respectively, about $5.36 \mu \mathrm{m}$ and about $5.80 \mu \mathrm{m}$ when immersing the substrates for $10 \mathrm{~s}$ and $20 \mathrm{~s}$ (Figures 3(c) and 3(d)). Tendency of the results is similar to what was reported by LLD theory $[11,12]$ on thicknesses of coating the Newtonian fluids.

Evaporation of the solvent was negligible since the substrate was spun immediately right after drawing the substrate out of the polymer solution bath. Spinning of the substrate eliminated polymer solution excess and blobs. In addition, spinning thinned the polymer solution coating on the substrate surface down further due to the radial outflow driven by the centrifugal force [17]. As a result of coating the substrates with a polymer solution having viscosity of $1100 \mathrm{mPa} \cdot \mathrm{s}$, immersion time of $20 \mathrm{~s}$, and withdrawal rate of $20 \mathrm{~mm} / \mathrm{s}$, the average coating thicknesses of the polymer films decreased, for example, from about $7.21 \mu \mathrm{m}$ to about $6.40 \mu \mathrm{m}$, Figures 4(a) and 4(b), at higher spin speeds of $100 \mathrm{rpm}$ and $500 \mathrm{rpm}$, respectively. Result trends are consistent with what is reported by Emslie et al. [18] on thicknesses of coating the Newtonian fluids.

When the solid volume of polymer particles in a continuous medium increases, viscosity of the polymer solution increases. High solid volume of polymer particles leads to high coating thickness [16]. At given conditions of withdrawal rate of $20 \mathrm{~mm} / \mathrm{s}$, spin speed of $500 \mathrm{rpm}$, and immersion time of $10 \mathrm{~s}$, for example, the average coating thicknesses of the polymer films increased from about $4.63 \mu \mathrm{m}$ to about $5.87 \mu \mathrm{m}$ with increasing viscosities from $900 \mathrm{mPa} \cdot \mathrm{s}$ to $1100 \mathrm{mPa} \cdot \mathrm{s}$ (Figures 5(a) and 5(b)).

Following the coating conditions, all measurement results and their average thicknesses are tabulated in Table 2.
TABLE 3: Estimated effect and coefficients for coating thickness (coded units).

\begin{tabular}{lccc}
\hline Term & Effect & Coefficient & $p$ value \\
\hline Constant & & 6.0362 & $0.000^{*}$ \\
$A$ & 1.6767 & 0.8383 & $0.000^{*}$ \\
$B$ & 0.7092 & 0.3546 & $0.000^{*}$ \\
$C$ & -0.3858 & -0.1929 & $0.000^{*}$ \\
$D$ & 0.6642 & 0.3321 & $0.000^{*}$ \\
$A B$ & 0.1767 & 0.0883 & $0.001^{*}$ \\
$A C$ & -0.1450 & -0.0725 & $0.007^{*}$ \\
$A D$ & 0.2033 & 0.1017 & $0.000^{*}$ \\
$B C$ & 0.0308 & 0.0154 & 0.546 \\
$B D$ & 0.0708 & 0.0354 & 0.170 \\
$C D$ & -0.0342 & -0.0171 & 0.504 \\
$A B C$ & 0.0250 & 0.0125 & 0.624 \\
$A B D$ & 0.0500 & 0.0250 & 0.330 \\
$A C D$ & -0.0150 & -0.0075 & 0.769 \\
$B C D$ & 0.1158 & 0.0579 & $0.028^{*}$ \\
$A B C D$ & 0.0517 & 0.0258 & 0.314 \\
Center point & & 0.0050 & 0.550 \\
\hline
\end{tabular}

$R^{2}=99.76 \%$; Adj. $R^{2}=96.76 \%$; $p$ value of $\mathrm{LOF}=0.65 ;{ }^{*}$ significant at significance level of less than 0.05 .

3.2. Statistical Analysis. Table 3 summarizes the results of the statistical analysis performed on experimental data. In particular, the table shows estimated effect, regression model coefficients, and corresponding $p$ values for each processing parameter and parameter combination. The $p$ value represents the significance level, probability to reject the null hypothesis, $H_{0}$, although it is true. A $p$ value smaller than 0.05 indicates that the model is significant with significance level of less than 0.05 .

From Table 3, three-way interaction effects of withdrawal rate, spin speed, and immersion time $(B C D)$ and two-way 
interaction effects of viscosity and withdrawal rate $(A B)$, viscosity and spin speed $(A C)$, and viscosity and immersion time $(A D)$ on the coating thickness are significant at a significance level of less than 0.05 . In addition, all main effects are significant with significance level of less than 0.001 . However, the effect of the center point is not significant with significance level of 0.05 . This implies that the curvature is not expected. The rest of the interaction effect terms are also not significant with significance level of 0.05 .

Values of the coefficient of determination $\left(R^{2}\right)$, the adjusted coefficient of determination (Adj. $R^{2}$ ), and the $p$ value of lack of fit (LOF) are used to evaluate the goodness of fit and adequacy of the model [13]. $R^{2}$ value of $97.76 \%$ indicates a very good fit, meaning that $97.76 \%$ of variation in coating thickness can be explained by the fitted model. The Adj. $R^{2}$ value indicates how $R^{2}$ value overestimates variation in the coating thickness when another predicted term is added in the model. The closer the Adj. $R^{2}$ value to the $R^{2}$ value, the higher the goodness of fit, meaning that an additional term in the model is significant and should be included. The $p$ value of lack of fit of 0.65 indicates that lack of fit is not significant, relative to the pure error with significance level of 0.05 . Thus, the model is adequate.

The empirical model in terms of coded parameters after excluding the insignificant terms for coating thickness is

$$
\begin{aligned}
Y= & 6.0362+0.8383 A+0.3546 B-0.1929 C \\
& +0.3321 D+0.0883 A B-0.0725 A C+0.1017 A D \\
& +0.0579 B C D
\end{aligned}
$$

The coefficient is exactly the quantitative measure of effect. The higher the coefficient, the stronger the effect of the parameter. The coefficient sign gives the direction of parameter effect. In (3), the positive sign of coefficients for main effects of viscosity $(A)$, withdrawal rate $(B)$, and immersion time $(D)$ indicates that an increase of each parameter in these terms leads to increased coating thickness. The negative sign of coefficients for spin speed $(C)$ term indicates that higher spin speeds lead to reduced coating thickness.

Two-way interaction effects between viscosity and immersion time $(A D)$ with a positive sign mean that a simultaneous increase or decrease of these two parameters leads to obtaining a thicker coating. This indicates synergistic effects of viscosity and immersion time on the coating thickness while the negative sign of two-way interaction effects between viscosity and spin speed $(A C)$ denotes an antagonistic effect.

Three-way interaction effects between withdrawal rate, spin speed, and immersion time $(B C D)$ with a positive sign also indicated a synergistic effect of these parameters on the coating thickness.

The transformation for each coded level, from (1), is represented by

$$
A_{\text {Coded }}=\frac{\left(A_{\text {Uncoded }}-1000\right)}{100}
$$

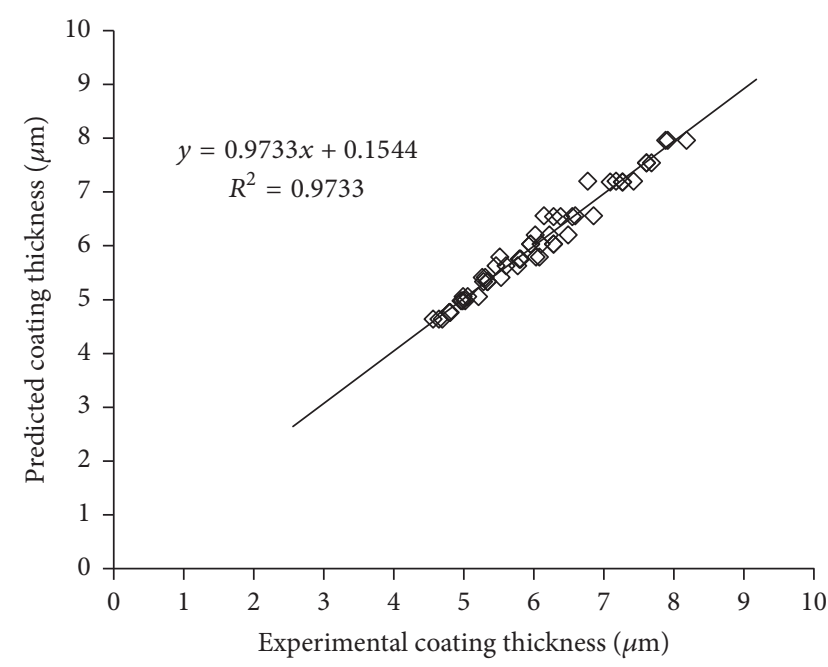

FIgURE 6: Comparison of predicted and experimental coating thicknesses.

$$
\begin{aligned}
B_{\text {Coded }} & =\frac{\left(B_{\text {Uncoded }}-22.5\right)}{2.5} \\
C_{\text {Coded }} & =\frac{\left(C_{\text {Uncoded }}-300\right)}{200} \\
D_{\text {Coded }} & =\frac{\left(D_{\text {Uncoded }}-15\right)}{5} .
\end{aligned}
$$

Using these equations, (3) can be adjusted for the uncoded level of each factor and expressed as

$$
\begin{aligned}
Y= & 1.3204-0.00153 A-0.10714 B+0.01048 C \\
& +0.01935 D+0.00035 A B-3.6 \times 10^{-6} A C \\
& +0.0002 A D-0.00035 B C-0.00695 B D \\
& -0.00052 C D+2.32 \times 10^{-5} B C D .
\end{aligned}
$$

Figure 6 shows the comparison between predicted values from (5) and the experimental values. The predicted model fits well with the experimental results and the error is about $0.12 \%$. However, it should be noted that the model could be applied only under the stated experimental boundary.

Figure 7 shows three-way interaction effect of withdrawal rates, spin speed, and immersion time $(B C D)$. Individually shown in Figures 7(a) and 7(b), two-way interaction effect plots of spin speed and immersion time $(C D)$ at withdrawal rates $(B)$ of $20 \mathrm{~mm} / \mathrm{s}$ and $25 \mathrm{~mm} / \mathrm{s}$, respectively, reveal nonparalleled, codirectional behaviors within the two parameters of spin speed and immersion time $(C D)$. Moreover, the twoway interaction effect plots of spin speed and immersion time $(C D)$ differ across levels of withdrawal rates $(B)$ demonstrating three-way interaction effect of withdrawal rates, spin speed, and immersion time $(B C D)$. Unlike Figure 7 , Figures $8(\mathrm{a}), 8(\mathrm{~b})$, and $8(\mathrm{c})$, respectively, show only twoway interaction effects of viscosity and withdrawal rate $(A B)$, 


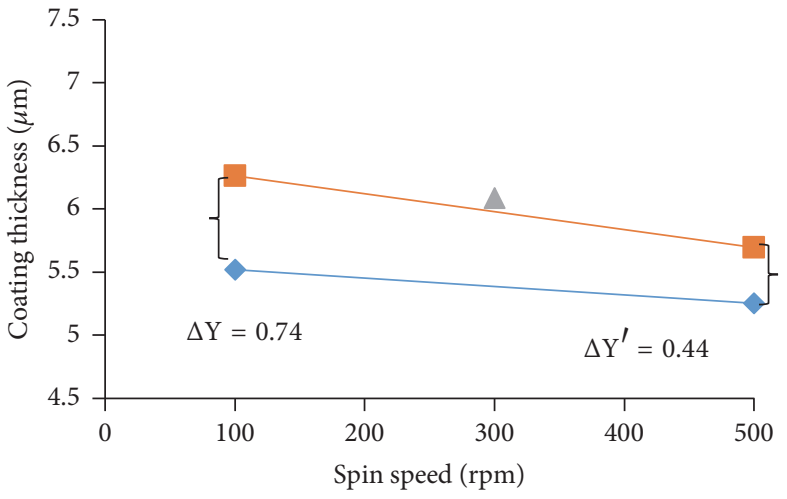

Immersion time

$\checkmark 10 \mathrm{~s}$

$20 \mathrm{~s}$

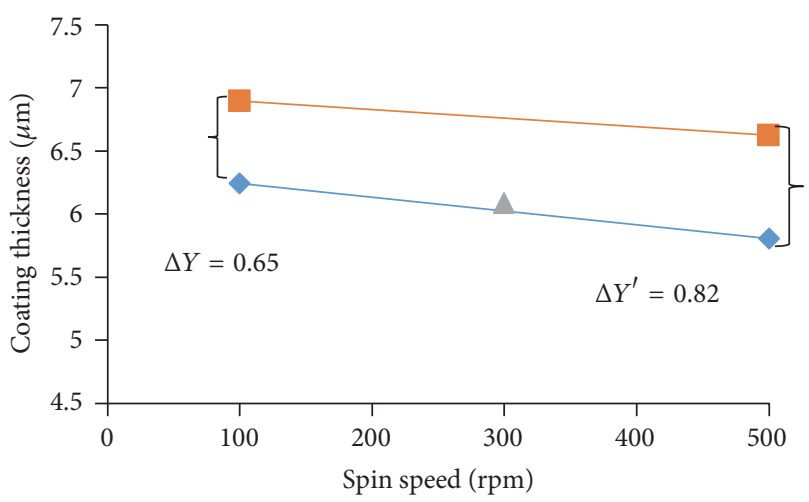

Immersion time

$-10 \mathrm{~s}$

$20 \mathrm{~s}$ $\triangle$ Center point

(b)

FIGURE 7: Interaction effect plots of spin speed and immersion time at (a) withdrawal rate of $20 \mathrm{~mm} / \mathrm{s}$ and (b) withdrawal rate of $25 \mathrm{~mm} / \mathrm{s}$.

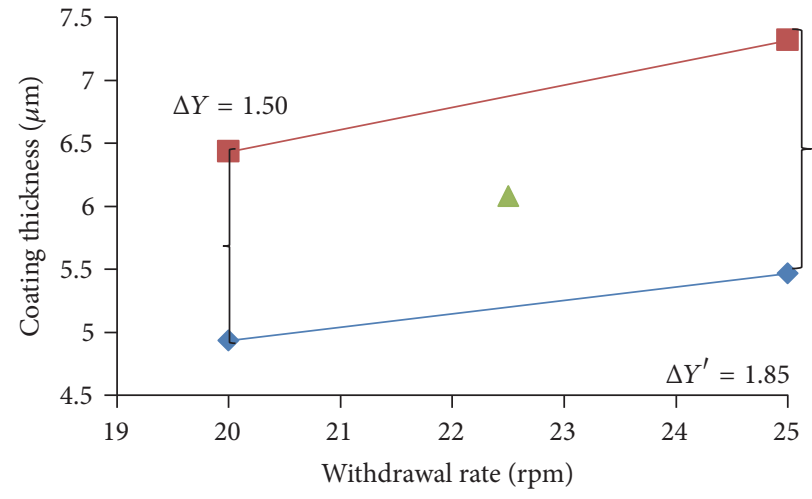

Viscosity

$\neg 900 \mathrm{cP}$

$-1100 \mathrm{cP}$

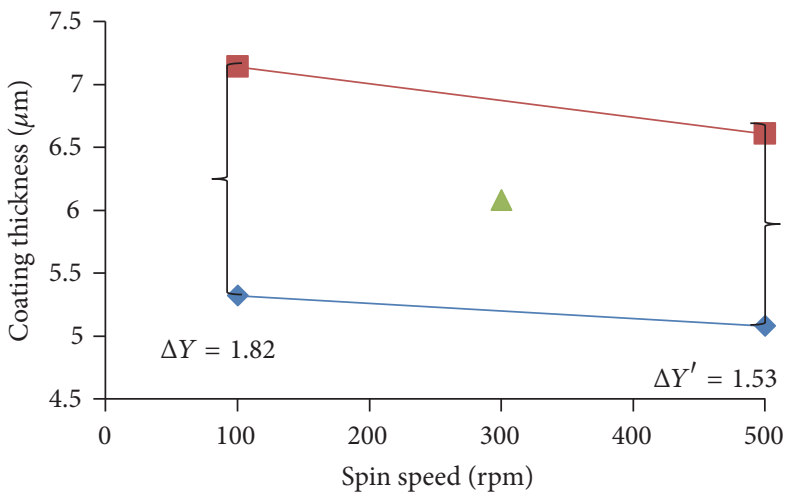

Viscosity

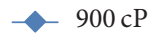

$1100 \mathrm{cP}$
Center point

(b)

(a)

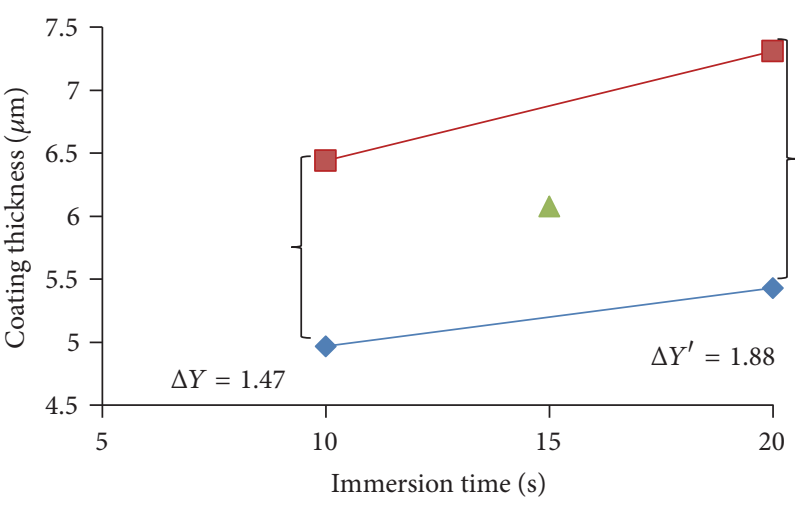

Viscosity

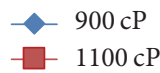

$\Delta$ Center point

(c)

Figure 8: Interaction effect plots of viscosity with (a) withdrawal velocity, (b) spin speed, and (c) immersion time for coating thickness. 


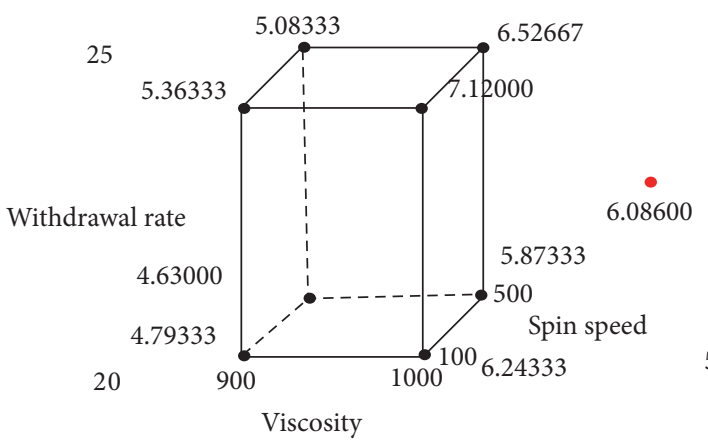

10

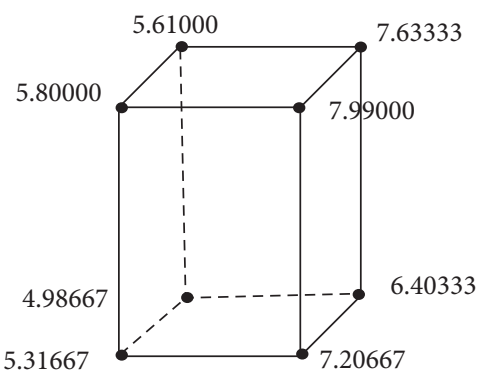

Immersion time

- Center point

- Factorial point

FIGURE 9: Cube plot for coating thickness.

viscosity and spin speed $(A C)$, and viscosity and immersion time $(A D)$ that are not significantly affected across levels of the remaining parameters.

Cube plot, Figure 9, displays average coating thicknesses of all conditions. The cube plot depicts the trend of increased coating thickness towards high viscosity, high withdrawal rate, long immersion time, and low spin speed. If the target of the coating thickness is set at about $5 \mu \mathrm{m}$, for example, the parameters should be set around the coating conditions following viscosity of $900 \mathrm{mPa} \cdot \mathrm{s}$, withdrawal rate of $25 \mathrm{~mm} / \mathrm{s}$, spin speed of $500 \mathrm{rpm}$, and immersion time of $10 \mathrm{~s}$. Substituting these coating parameters in the statistical model, the calculated value is $5.06 \mu \mathrm{m}$. The value is approximately the same as the experimental coating thickness of $5.08 \mu \mathrm{m}$.

In order to assess the reliability of the value predicted by the empirical statistical model, an actual experiment was carried out based on the coating condition: viscosity of $1100 \mathrm{mPa} \cdot \mathrm{s}$, withdrawal rate of $20 \mathrm{~mm} / \mathrm{s}$, spin speed of $100 \mathrm{rpm}$, and immersion time of $10 \mathrm{~s}$. Using (5), the predicted thickness is $6.12 \mu \mathrm{m}$. The experimental thickness is $6.24 \mu \mathrm{m}$. The result shows that the modeling approach presented in this study can accurately predict the coating thickness.

\section{Conclusion}

This study analyzed the effects of the coating parameters, namely, viscosity, withdrawal rate, spin speed, and immersion time, on the coating thickness by using a $2^{4}$ factorial design with a center point. The external surface of the hollow tube with the outer diameter of $450 \mu \mathrm{m}$ was successfully coated with colloidal PTFE dispersion by using the dip-spin coating equipment. The tube end was clear of polymer blobs and the size of the original inner tube diameter, $260 \mu \mathrm{m}$, was still maintained. The statistical model is expressed with only significant terms using significance level of less than 0.05 . The significant interaction effects on the coating thickness included three-way interactions of withdrawal rate, spin speed, and immersion time and two-way interactions of viscosity and withdrawal rate, viscosity and spin speed, and viscosity and immersion time. The statistical model accuracy is verified by comparing the predicted values with the experimental results. The predicted values are fitted with the experimental results. Cube plot analysis indicates higher coating thickness with increased viscosity, higher withdrawal rate, increased immersion time, and decreased spin speed. Verification experiment shows that the statistical model developed can be used for PTFE coating thickness.

\section{Conflicts of Interest}

The authors declare that they have no conflicts of interest.

\section{Acknowledgments}

The authors gratefully acknowledge the financial support of this work provided by the National Research Council of Thailand (NRCT) and Thailand's Health Systems Research Institute (HSRI).

\section{References}

[1] H. I. Rowan, “Thermoplastic Fluoropolymers of engineering plastics," in Engineered Materials Handbook, pp. 115-119, ASM International, Metals Park, Ohio, USA, 1988.

[2] L. W. McKeen, Fluorinated Coatings and Finishes Handbook, William Andrew Publishing, New York, NY, USA, 2006.

[3] D. A. White and J. A. Tallmadge, "Theory of drag out of liquids on flat plates," Chemical Engineering Science, vol. 20, no. 1, pp. 33-37, 1965.

[4] P. Yimsiri and M. R. MacKley, "Spin and dip coating of light-emitting polymer solutions: matching experiment with modelling," Chemical Engineering Science, vol. 61, no. 11, pp. 3496-3505, 2006.

[5] H.-W. Fang, K.-Y. Li, T.-L. Su et al., "Dip coating assisted polylactic acid deposition on steel surface: film thickness affected by drag force and gravity," Materials Letters, vol. 62, no. 21-22, pp. 3739-3741, 2008. 
[6] C. Gao, Y. C. Lee, J. Chao, and M. Russak, "Dip-coating of ultrathin liquid lubricant and its control for thin-film magnetic hard disks," IEEE Transactions on Magnetics, vol. 31, no. 6, pp. 29822984, 1995.

[7] C. J. Brinker, G. C. Frye, A. J. Hurd, and C. S. Ashley, "Fundamentals of sol-gel dip coating," Thin Solid Films, vol. 201, no. 1, pp. 97-108, 1991.

[8] I. Strawbridge and P. F. James, "The factors affecting the thickness of sol-gel derived silica coatings prepared by dipping," Journal of Non-Crystalline Solids, vol. 86, no. 3, pp. 381-393, 1986.

[9] C. Jing, X. Zhao, and H. Tao, "An approach to predict the solid film thickness possibly yielded from an alumina sol-gel liquid film," Surface and Coatings Technology, vol. 201, no. 6, pp. 26552661, 2006.

[10] L. J. Crawford and N. R. Edmonds, "Calculation of film thickness for dip coated antireflective films," Thin Solid Films, vol. 515, no. 3, pp. 907-910, 2006.

[11] L. Landau and B. Levice, "Dragging of a liquid by a moving plat," Acta Physicochimica U.R.S.S, vol. 17, no. 1, pp. 1942-1954, 1942.

[12] B. V. Derjaguin, "On the thickness of the liquid film adhering to the wall of a vessel after emptying," Acta Physicochimica U.R.S.S, vol. 20, 1943.

[13] D. C. Montgomery, Design and Analysis of Experiments, John Wiley \& Sons, New York, NY, USA, 2013.

[14] ASTM International, "Test methods for rheological properties of non-Newtonian materials by rotational (Brookfield type) viscometer," ASTM D2196-10, 2010.

[15] D. Stoye and W. Freitag, Paints, Coatings and Solvents, WileyVCH, New York, NY, USA, 2001.

[16] W. Wick Zeno Jr., N. Jones Frank, P. S. Peter, and A. Wicks Doulas, Organic Coatings, Science and Technology, WileyInterscience, New York, NY, USA, 2007.

[17] D. Meyerhofer, "Characteristics of resist films produced by spinning," Journal of Applied Physics, vol. 49, no. 7, pp. 39933997, 1978.

[18] A. G. Emslie, F. T. Bonner, and L. G. Peck, "Flow of a viscous liquid on a rotating disk," Journal of Applied Physics, vol. 29, no. 5, pp. 858-862, 1958. 

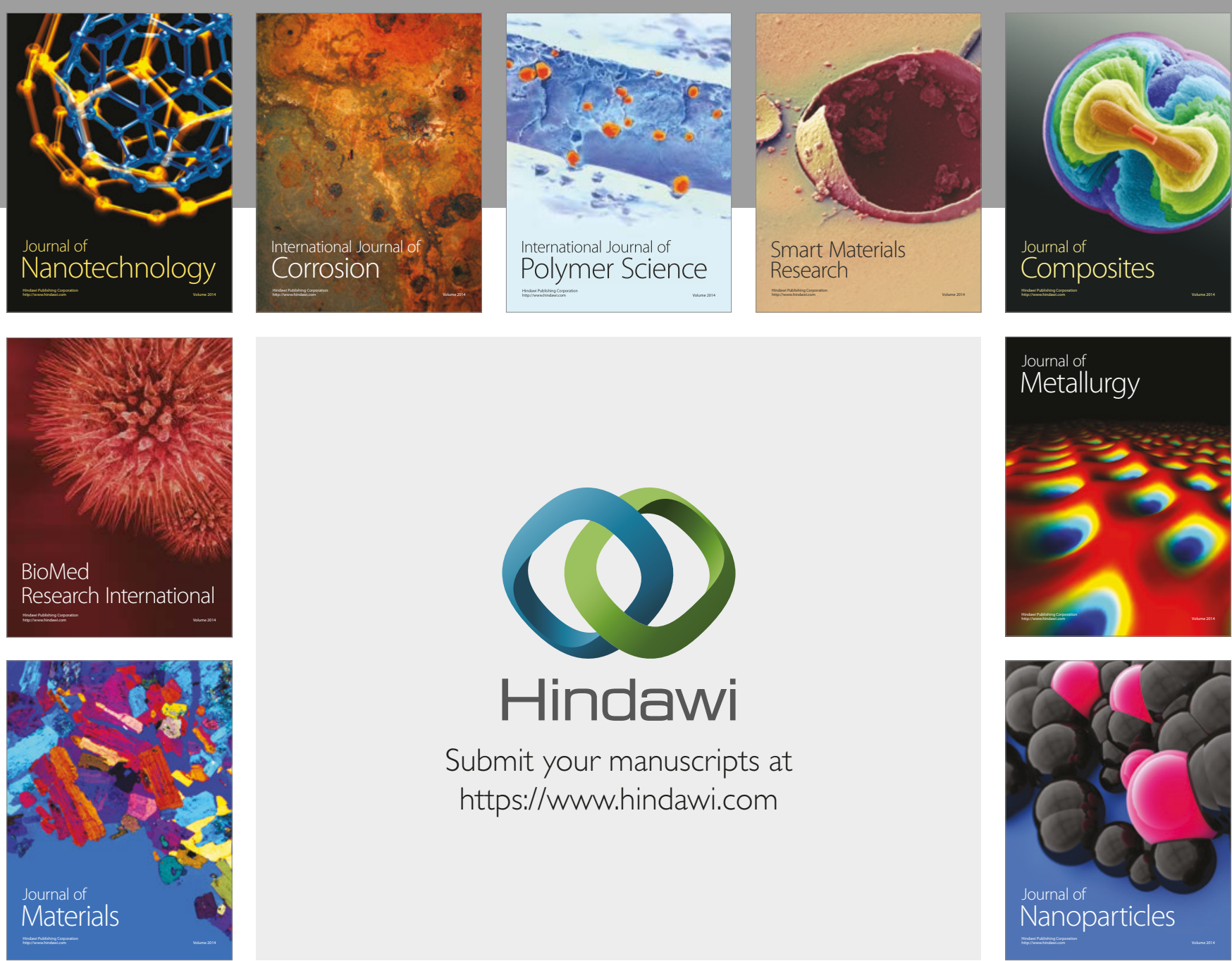

\section{Hindawi}

Submit your manuscripts at

https://www.hindawi.com
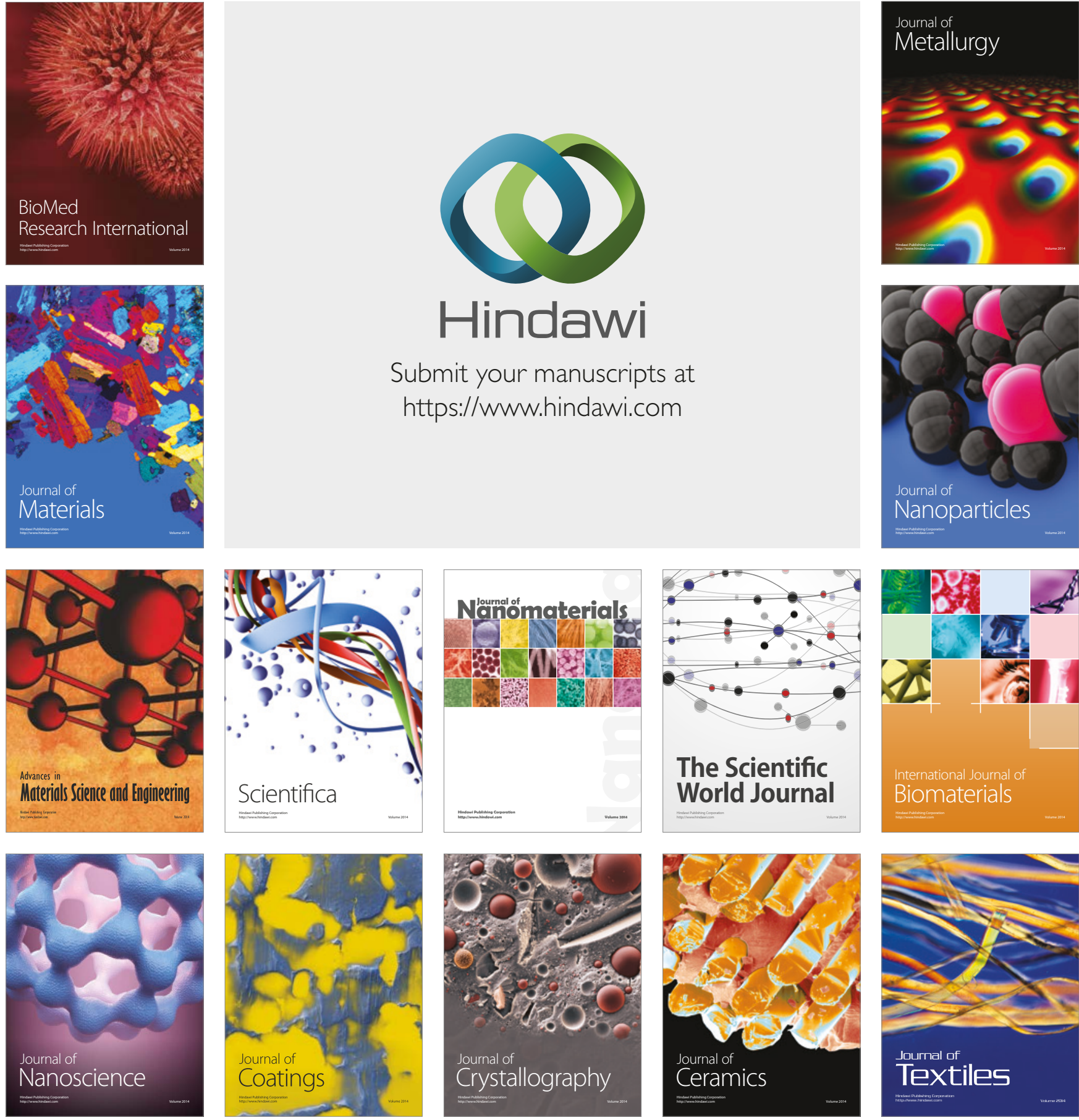

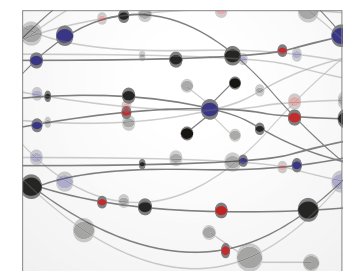

The Scientific World Journal
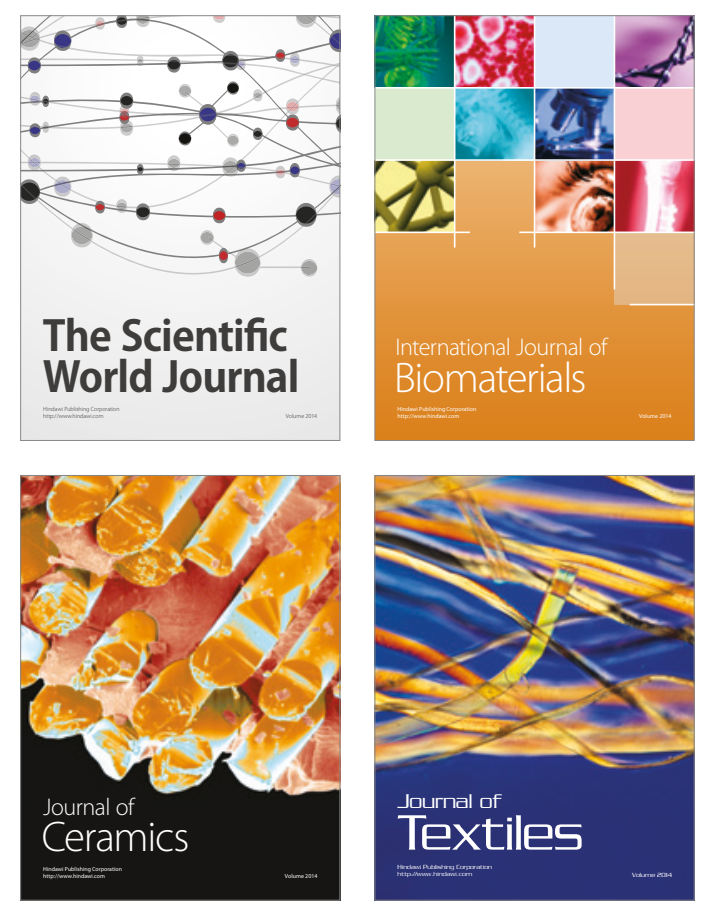